\title{
Review of Heavy-Duty Engine Combustion Research at Sandia National Laboratories
}

Robert W. Carling

Sandia National Lab.

Gurpreet Singh

Dept. of Energy 
SAE routinely stocks printed papers for a period of three years following date of publication. Direct your orders to SAE Customer Sales and Satisfaction Department.

Quantity reprint rates can be obtained from the Customer Sales and Satisfaction Department.

To request permission to reprint a technical paper or permission to use copyrighted SAE publications in other works, contact the SAE Publications Group.

This article was prepared as an account of work sponsored by an agency of the United States

Government. Neither the United States Government nor any agency thereof, nor any of their employees, makes any warranty, express or implied, or assumes any legal liability or responsibility for the accuracy, completeness, or usefulness of any information, apparatus, product, or process disclosed, or represents that its use would not infringe privately owned rights. Reference herein to any specific commercial product, process, or service by trade name, trademark, manufacturer, or otherwise does not necessarily constitute or imply its endorsement, recommendation, or favoring by the United States Government or any agency thereof. The views and opinions of authors expressed herein do not necessarily state or reflect those of the United States Government or any agency thereof.

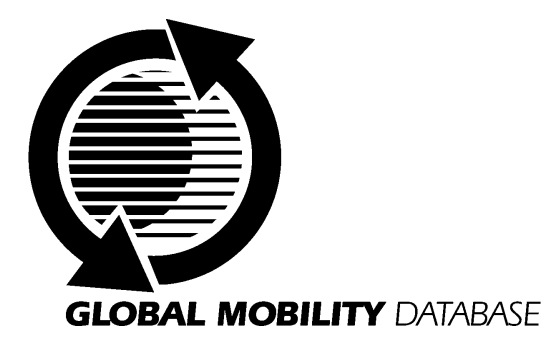

All SAE papers, standards, and selected books are abstracted and indexed in the Global Mobility Database

ISSN 0148-7191

No copyright is asserted in the works of U.S. Government employees.

Positions and opinions advanced in this paper are those of the author(s) and not necessarily those of SAE. The author is solely responsible for the content of the paper. A process is available by which discussions will be printed with the paper if it is published in SAE Transactions. For permission to publish this paper in full or in part, contact the SAE Publications Group.

Persons wishing to submit papers to be considered for presentation or publication through SAE should send the manuscript or a 300 word abstract of a proposed manuscript to: Secretary, Engineering Meetings Board, SAE.

\section{Printed in USA}




\section{Review of Heavy-Duty Engine Combustion Research at Sandia National Laboratories}

Robert W. Carling

Sandia National Lab.

Gurpreet Singh

Dept. of Energy

No copyright is asserted in the works of U.S. Government employees

\begin{abstract}
The objectives of this paper are to describe the research efforts in diesel engine combustion at Sandia National Laboratories' Combustion Research Facility and to provide recent experimental results. We have four diesel engine experiments supported by the Department of Energy, Office of Heavy Vehicle Technologies: a one-cylinder version of a Cummins heavy-duty engine, a diesel simulation facility, a one-cylinder Caterpillar engine to evaluate combustion of alternative fuels, and a homogeneous-charge, compression-ignition $(\mathrm{HCCl})$ engine facility is under development. Recent experimental results to be discussed are: the effects of injection timing and diluent addition on late-combustion soot burnout, dieselspray ignition and premixed-burn behavior, a comparison of the combustion characteristics of M85 (a mixture of $85 \%$ methanol and $15 \%$ gasoline) and DF2 (\#2 diesel reference fuel), and a description of our $\mathrm{HCCl}$ experimental program and modeling work.
\end{abstract}

\section{INTRODUCTION}

Diesel engines play an important role in the U.S. economy accounting for nearly all of the engines in the Class 7 and 8 categories, and a substantial fraction of the engines in lighter trucks. The Engine Combustion Department of Sandia National Laboratories' Combustion Research Facility has four laboratories supported via the Department of Energy Office of Heavy Vehicle Transportation Technologies: a one-cylinder version of a Cummins heavy-duty engine, a diesel simulation facility, a one-cylinder Caterpillar engine to evaluate combustion of alternative fuels, and a homogeneous-charge, compression-ignition $(\mathrm{HCCl})$ engine that is currently being developed. The research conducted in these laboratories supports the heavy-duty diesel engine manufacturers by providing fundamental understanding of the combustion processes that control engine efficiency and emissions formation.
This paper is intended to briefly describe the facilities and projects and to recent results. It is divided into four sections focusing on each of the four experiments. This paper draws from several recent publications and provides a current summary of our progress in understanding in-cylinder diesel combustion.

\section{SPECIFIC EXPERIMENTS}

Each section will discuss a specific experiment and recent results.

SANDIA/CUMMINS HEAVY-DUTY ENGINE - Diesel engine design continues to be driven by the need to improve performance while at the same time achieving further reductions in emissions. The development of new designs to accomplish these goals requires an understanding of how the emissions are produced in the engine. Laserimaging diagnostics are uniquely capable of providing this information; however, previous laser-imaging studies have generally focused on the early and middle stages of diesel combustion. Prior to the investigation reported here, there had been little detailed information on the late stages and the mechanism whereby soot escapes combustion to become a tail-pipe emission.

For this investigation, we first developed the capability of simultaneously imaging the $\mathrm{OH}$-radical and soot distributions in the diesel engine with planar laser-induced fluorescence (OH-PLIF) and planar laser-induced incandescence (PLII), respectively. After initial development, the technique was demonstrated as a means of studying the late-combustion soot burnout for a base operating condition corresponding to a medium speed (1200 rpm) and medium-low load. The work was then extended to include investigations of a higher-speed (1680 rpm), higher-load condition and the effects of simulated EGR (exhaust gas recirculation) by the addition of nitrogen diluent [1]. 
Experimental hardware - The engine we are using is a one-cylinder version of a 4-stroke diesel engine based on a Cummins $\mathrm{N}$-series production engine. The $\mathrm{N}$-series engine is typical of heavy-duty size-class diesel engines (Class $7 \&$ 8), with a bore of $140 \mathrm{~mm}$ and a stroke of 152 $\mathrm{mm}$. These dimensions are retained in the opticalaccess engine, and a production Cummins $\mathrm{N}$-series cylinder head is used so that the production engine intake port geometry is also preserved. The design of this engine utilizes a classic extended piston with pistoncrown window. This piston-crown window provides a full view of the combustion bowl. A complete description of this engine may be found in Ref. [2].

A dual-laser, dual-camera system, shown in fig. 1 was used to obtain the simultaneous "single-shot" images.

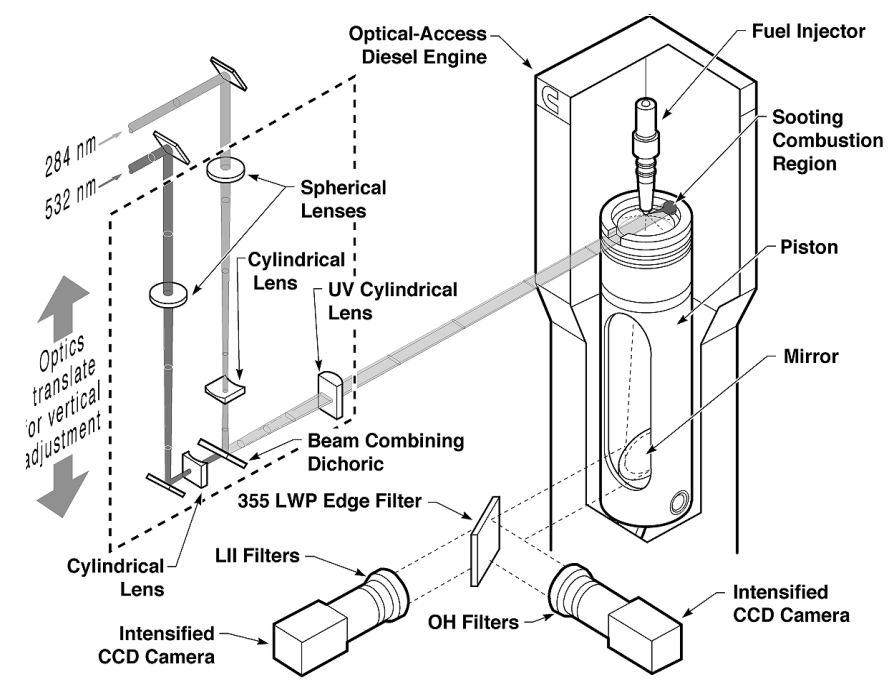

Figure 1. Schematic of the optical-access diesel engine showing the optical setup for simultaneous OH-PLIF and PLII-soot imaging. Only one of the 7 fuel jets is shown.

The two laser beams were combined into overlapping laser sheets approximately $25 \mathrm{~mm}$ wide and $0.2 \mathrm{~mm}$ and $0.3 \mathrm{~mm}$ thick for the PLIF and PLII lasers, respectively. After being combined, the beams were directed into the combustion chamber through one of the windows at the top of the cylinder wall which was opposite the region of the imaged fuel jet. The fuel jet opposite the imaged jet was removed to minimize laser attenuation, resulting in a 7-hole injector, rather than the production 8-hole unit. In addition, the piston bowl rim was cut out inline with the entrance window and "missing" jet to allow laser access to the combustion chamber when the piston is near top dead center. The simultaneous OH-PLIF and PLII-soot images were acquired through the piston-crown window, as also shown in Fig. 1.

Recent results - The well known $\mathrm{NO}_{x}$-particulate tradeoff in diesel engines typically becomes more severe when fuel loading and engine speed are increased. As a result, particulate emissions can be difficult to control when injection timing is retarded to reduce $\mathrm{NO}_{x}$ at highload, high-speed conditions. Particulate emissions can also become problematic when exhaust gas recirculation (EGR) is used for $\mathrm{NO}_{x}$ control

Measurements were made at a $1680 \mathrm{rpm}$, high-load operating condition. Simultaneous images, obtained with the dual-laser, dual-camera system, showed the spatial relationship between the remaining hot reaction zones and the remaining bulk-gas soot during the final stages of the combustion event. The data show significant variations in the late-combustion burnout of soot in the bulk gases (i.e., away from the combustion-chamber walls) as timing is retarded and $N_{2}$ diluent (simulated EGR) is added, as is done in production engines to control $\mathrm{NO}_{X}$ emissions.

Several of the conclusions from this recent study follow:

- For a medium-low load, medium speed ( $C / F=65$, $1200 \mathrm{rpm}$ ) condition, all the bulk-gas soot appears to burnout well before the last $\mathrm{OH}$ disappears, for both normal and $11.5^{\circ}$-retarded timing. At a higher-load and higher-speed ( $\mathrm{C} / \mathrm{F}=31,1680 \mathrm{rpm})$ condition, all the bulk-gas soot burns out for normal injection timing; however, for $10.5^{\circ}$-retarded timing, significant bulk-gas soot remains even after exhaust valve opening (EVO).

- With the addition of $10 \% \mathrm{~N}_{2}$ diluent (simulated $\mathrm{EGR}$ ), at the high-load, 1680-rpm condition, significant bulkgas soot remains after EVO even with normal timing. The combination of $10 \% \quad \mathrm{~N}_{2}$ diluent and $10.5^{\circ}$ retarded timing greatly diminishes the bulk-gas soot burnout.

- In contrast with image data, the apparent heat release rate curves show little difference in the pressure-indicated heat release when $10 \% \mathrm{~N}_{2}$ is added, for either injection timing. Thus, changes in combustion that are not detectable with pressure measurements can have a large impact on emissions.

- Exhaust-gas soot measurements were also made, and a strong correlation was found between changes in the bulk-gas soot burnout and engine-out soot measurements. This correlation suggests that incomplete burnout in the bulk gas is a major contributor to the increased soot emissions typically observed with timing retard and diluent addition.

DIESEL COMBUSTION SIMULATION FACILITY - Diesel spray autoignition is central to the operation of a diesel engine. The autoignition quality of diesel fuel, rated in terms of a cetane number, is an important factor in determining diesel engine performance and emissions. Understanding the diesel ignition process in greater detail will aid the development of advanced diesel engines, fuels/additives that enhance the performance and emissions characteristics of diesels, and improved multi-dimensional diesel combustion models. 
In recent experiments we have studied the diesel spray autoignition process in more detail. In general, the information presented supports the results of recent investigations of the timing and spatial evolution of events during ignition and the premixed burn in a heavy-duty diesel engine by Dec and Espey [3,4]. Similarities and differences noted between the two investigations are discussed more completely in another publication [5]. The differences lead to additional insight that has implications for modeling of diesel sprays. A summary of the findings is presented here.

Experimental hardware - The diesel spray ignition investigations discussed in the following section were conducted in a constant-volume combustion vessel with extensive optical access. This facility is discussed in detail in other publications $[5,6]$. Fuels were injected with an electronically controlled, common-rail injector using a single hole orifice, i.e., a single-spray plume was considered. Simultaneously measured pressure histories, total natural light emission histories, and line-of-sight integrated luminosity images were used to investigate the ignition and premixed-burn phases. These were coupled as needed with previous gas-phase penetration [7], liquid-phase fuel penetration [8], and spreading angle measurements [9] for sprays obtained under the same conditions.

Recent results - Ambient gas conditions in the combustion vessel included temperatures from 800 to $1100 \mathrm{~K}$ at a density of $14.8 \mathrm{~kg} / \mathrm{m}^{3}$, and densities from 7.27 to 45.0 $\mathrm{kg} / \mathrm{m}^{3}$ at a temperature of $1000 \mathrm{~K}$. These gas temperature and density ranges include top-dead-center temperatures and densities typical of those in heavy-duty and light-duty direct-injection diesel engines. The fuel used was a ternary blend of single-component fuels with a cetane number of 45 .

For gas temperatures and densities characteristic of a moderate load heavy-duty diesel condition, a two-stage ignition process was clearly observed. As temperature and density decreased (approached lighter load and cold-start conditions), the duration of both stages of the ignition process lengthened and the location of ignition moved further downstream, as well as further from the liquid fuel jet. The ignition region also became leaner, but was still fuel rich. Other features of the ignition process remained the same as for the moderate load condition.

As temperature and density increased (approached those typical of high engine loads), the general character of the ignition process began to change. The duration of the stages of ignition significantly shortened, resulting in a rapid, seamless transition temporally through the stages of ignition. The spatial location of ignition moved upstream and surrounded the central liquid-fuel containing region of the spray, extending upstream along its sides. These side regions are characterized by high shear and radial gradients of temperature and concentration, as opposed to the more uniformly mixed downstream ignition regions noted for longer ignition delays.
The average equivalence ratio also became richer in the igniting region.

SANDIA/CATERPILLAR ENGINE - The effects of fuel formulation on the combustion process in diesel engines is as yet largely unexplored. Recent research has shown that alternative fuels can significantly reduce soot and $\mathrm{NO}_{x}$ emissions simultaneously, while preserving the high efficiency and power density of the diesel-cycle engine. In addition, many alternative fuels are renewable, so they have the potential to reduce petroleum imports as well as net greenhouse gas emissions.

Ongoing optical-engine research at Sandia and elsewhere has shed considerable light on the in-cylinder combustion processes for standard diesel fuel. However, many practical alternative fuels have vastly different physical and chemical properties, and the nature of their combustion and emissions-formation processes remains largely a mystery. Experiments that probe the inter-relationships between fuels and in-cylinder processes are essential to determine what types of molecules are ideally suited for diesel-cycle engines and why. The enhanced fundamental under-standing provided by such experiments will facilitate the identification of more "optimal" fuel formulations for the ultra-clean, high-efficient engines of the future.

Experimental hardware - The experimental optical engine for this project is similar to the one-cylinder San$\mathrm{dia} /$ Cummins engine described above but derived from a one-cylinder, Caterpillar 1.7- liter engine. A schematic of the optical engine is shown in Fig. 2.

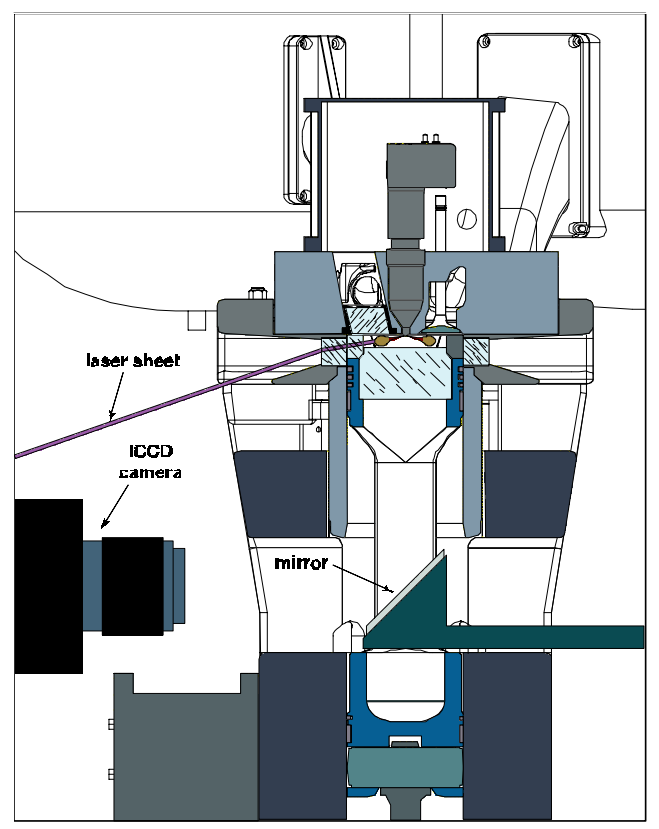

Figure 2. Cutaway view of a Caterpillar one-cylinder engine. Optical accessibility is similar to that in the Sandia/Cummins engine(shown in fig. 1).

Recent results - Experiments have been conducted comparing combustion characteristics of \#2 diesel fuel (DF2) with M85 (a mixture of $85 \%$ methanol with 15\% 
non-oxy-genated gasoline). The engine was run at a 1200 rpm low-load operating condition that had been determined to produce lower efficiency and higher unburned hydrocarbon emissions during tests with methanol fueling. The timing was set for maximum indicated torque. A sequence of direct luminosity images was acquired through the quartz piston top.

The following is a summary of our observations in the comparison of DF2 and M85:

- With DF2, all jets autoignite more or less simultaneously and burn independently of adjacent jets, yielding a symmetrical combustion pattern and the classic diesel heat release curve showing a single premixed burn spike followed by the mixing-controlled burn.

- M85 combustion has a totally different character than DF2. Autoignition does not occur naturally with M85 at this typical diesel-engine operating condition. Rather, ignition must be induced by a glow plug because the fuel has a cetane number of ten. The flame must then propagate to adjacent jets in order to traverse the combustion chamber.

- Heat release curves look much different for M85 compared to DF2. They show multiple spikes superimposed on a smooth baseline that appears like a spark-ignited engine heat-release plot. Based on the corresponding diesel luminosity images, it is our belief that the spikes are due to premixed burning each time the flame ignites the next adjacent jet, but this hypothesis remains to be verified.

- Minimal luminosity is observed from the region of the combustion chamber opposite the glow plug either because the jet directly opposite the glow plug does not ignite, or because it has had such a long time to premix before it ignites that it burns in a fuel-lean, non-sooting mode.

- For both M85 and DF2, the majority of the direct luminosity signal comes from incandescence of soot. The magnitude of the diesel luminosity from M85 is at least three orders of magnitude less than that of the DF2 reference fuel. This is mostly attributable to the reduced production of soot when using M85, which is highly oxygenated ( $43 \%$ by mass).

HCCI ENGINE - A new project has been started examining homogeneous-charge, compression-ignition ( $\mathrm{HCCl}$ ) engine combustion. $\mathrm{HCCl}$ offers good potential for verylow emissions and diesel-like efficiencies. It is not a new idea but one that is being considered more seriously because of Tier II emission standards. The concept is to well-mix fuel and air and compress the mixture until a volumetric reaction occurs. This volumetric reaction of fuel and air can be operated under lean (or very dilute with EGR) conditions resulting in very-low emissions. Efficiencies can be very high since the engine has a diesellike compression ratio, no throttle losses, and the heat release is closer to constant volume than a diesel engine. The challenges for an $\mathrm{HCCl}$ engine are the control of the start of reaction, the rate of heat release, and unburned hydrocarbon emissions. Our program is to provide the science base understanding of $\mathrm{HCCl}$ engine combustion and has both experimental and modeling components.

Experimental hardware - The laboratory is being assembled at this time. We will have two Cummins B-series engines in which to conduct experiments; one an allmetal engine and another an optical engine. In each engine only one cylinder will be used. We are also planning on a variable valve timing system to enable flexibility in changing compression ratios.

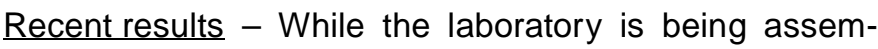
bled, we have been conducting kinetic-rate modeling to provide insight into the parameters controlling ignition timing in an $\mathrm{HCCl}$ engine and the sensitivity of ignition to changes in operating conditions. The CHEMKIN code has been used in the modeling with full chemistry for nheptane and iso-octane (obtained from Lawrence Livermore National Laboratory). The code allows time-varying compression of the reactants, which gives a good representation of $\mathrm{HCCl}$.

In this computational study, we have investigated the effects of compression ratio, intake temperature, fuel loading, engine speed, and the addition of EGR gases on $\mathrm{HCCl}$ ignition for both diesel fuel (n-heptane, $\mathrm{CN}=56$ ) and gasoline (iso-octane, $\mathrm{ON}=100$ ) surrogates [10]. The effects of compression ratio and fuel loading are summarized in the following examples.
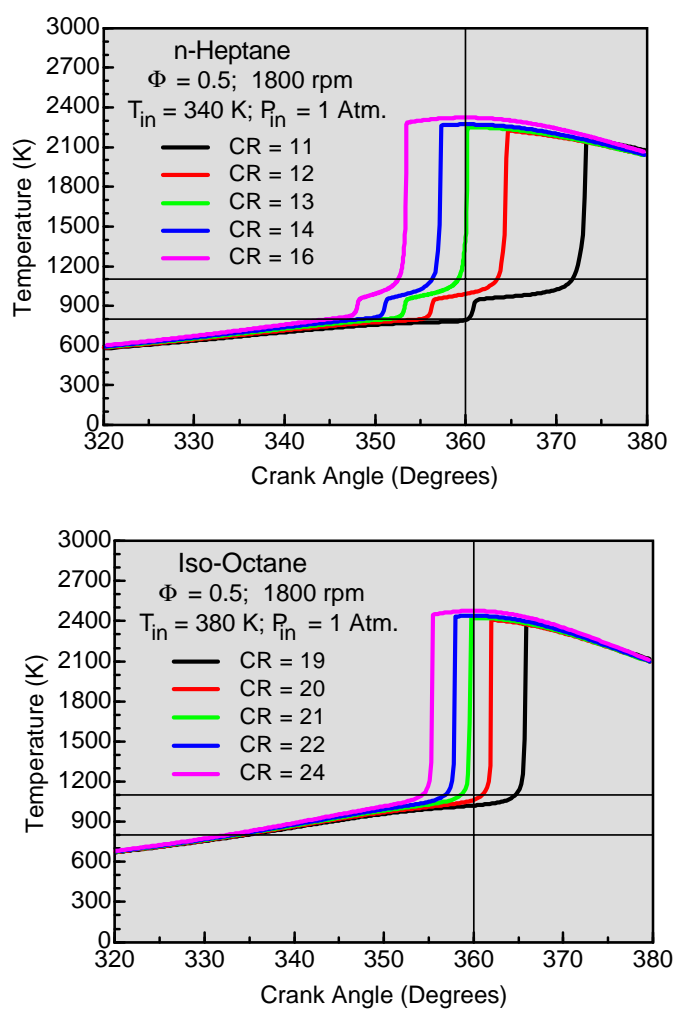

Figure 3. The Effect of varying compression ratio (CR) on ignition characteristics for a) $n$-heptane and b) iso-octane. 
Figure 3a, shows that the diesel surrogate has a 2-stage ignition reaction with the first stage taking place at a fairly low temperature of about $800 \mathrm{~K}$. This 2-stage chemistry prevents the use of higher diesel-like compression ratios. However, for the gasoline surrogate shown in Fig. 3b, the low-temperature chemistry is minimal with no evidence of a 2-step reaction and high, diesel-like compression ratios are allowed.
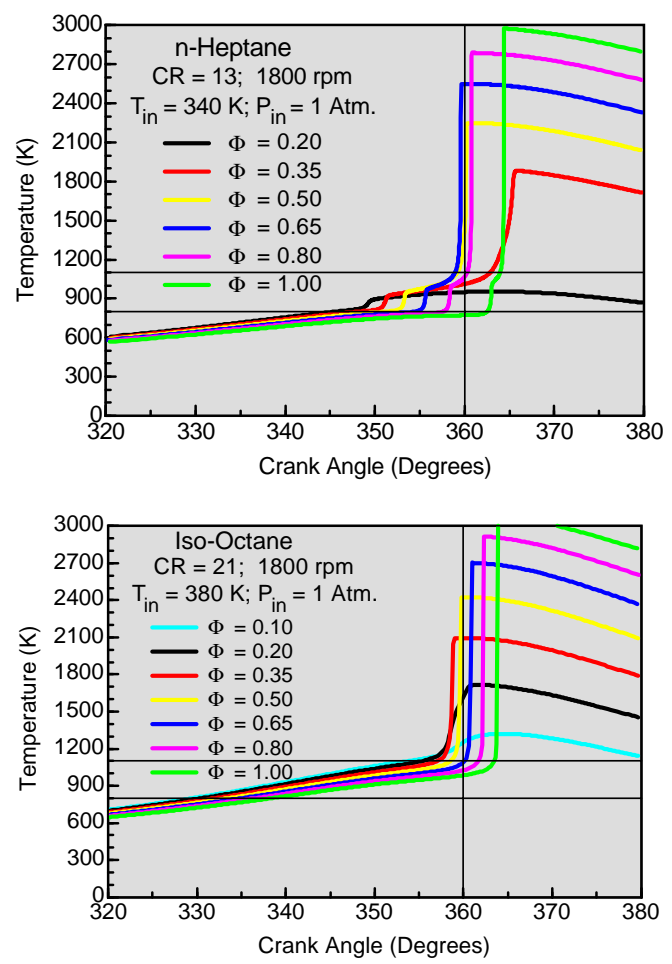

Figure 4. The effect of equivalence ratio (f) on ignition characteristics for a) n-heptane and b) isooctane.

The equivalence ratio was also varied, and the results are shown in Fig. 4. The data in Fig. 4a illustrate that ignition timing of the diesel-fuel surrogate is sensitive to fuel-air mixture due to active low-temperature chemistry. Also, the lower volatility of diesel fuel will make it harder to develop a uniform mixture in the engine. In contrast, ignition timing of the gasoline surrogate (Fig. 4b) is less sensitive to changes in the fuel-air mixture due to its single-stage ignition and lack of significant low-temperature chemistry. In addition, the high volatility of gasoline-like fuel will make it easier to develop a uniform mixture. The consequence is the potential for simpler combustion-control strategies and higher efficiencies with a gasoline-like fuel.

\section{ACKNOWLEDGMENTS}

This work was performed at the Combustion Research Facility, Sandia National Laboratories, Livermore, CA. Funding support was provided by the U.S. Department of Energy, Office of Heavy Vehicle Technologies. The authors would also like to express their appreciation to the principal investigators of the projects described above: John Dec (Sandia/Cummins and HCCl engines), Dennis Siebers (diesel combustion simulation vessel), and Chuck Mueller (Sandia/Caterpillar engine).

\section{REFERENCES}

1. Dec, J. E. and Kelly-Zion, P., "The Effects of Injection Timing and Diluent Addition on Late-Combustion Soot Burnout in a DI Diesel Engine Based on Simultaneous 2-D Imaging of $\mathrm{OH}$ and Soot, SAE paper no. 2000-01-0238, 2000.

2. Espey, C. and Dec, J. E., "Diesel Engine Combustion Studies in a Newly Designed Optical-Access Engine Using High-Speed Visualization and 2-D Laser Imaging," SAE Transactions, Vol. 102, Sec. 4, pp. 703723, paper no. 930971, 1993.

3. Dec, J. E. and Espey, C., "Ignition and Early Soot Formation in a D.I. Diesel Engine Using Multiple 2-D Imaging Diagnostics," SAE Transactions, Vol. 104, Sec. 3, pp. 853-875, 1995.

4. Dec, J. E. and Espey, C., "Chemiluminescence Imaging of Autoignition in a DI Diesel Engine," SAE Transactions (in press), SAE 982685, 1998.

5. Higgins, B., Siebers, D., and Aradi, A., "Diesel-Spray Ignition and Premixed-Burn Behavior," SAE paper no. 2000-01-0940, 2000.

6. Siebers, D. L., "Liquid-Phase Fuel Penetration in Diesel Sprays," SAE paper 980809, 1998.

7. Naber, J.D. and Siebers, D.L., "Effects of Gas Density and Vaporization on Penetration and Dispersion of Diesel Sprays," SAE Transactions, Vol. 105, Sec. 3, pp. 82-111, 1996.

8. Siebers, D.L., "Liquid-Phase Fuel Penetration in Diesel Sprays," SAE Transactions, Vol. 10, Sec. 3, pp. 1205-1227, 1998.

9. Siebers, D.L., "Scaling Liquid-Phase Fuel Penetration in Diesel Sprays Based on Mixing-Limited Vaporization," SAE 1999-01-0528.

10. Kelly-Zion, P. L. and Dec, J. E., "A Computational Study of the Effect of Fuel Type on Ignition Time in $\mathrm{HCCl}$ Engines," to be presented at and published in the proceedings of the $28^{\text {th }}$ Symposium (International) on Combustion, July \& August, 2000. 